\title{
Quantitative Voltage Control of Large-scale Wind Base
}

\author{
Zhengjun Xing ${ }^{1,}$, , Xiangyu Kong ${ }^{1, b}$, Fei Huang ${ }^{2, c}$ \\ ${ }^{1}$ School of Electrical and Electronic Engineering, North China Electric Power University, Beijing \\ 102206, China \\ ${ }^{2}$ School of Control and Computer Engineering, North China Electric Power University, Beijing \\ 102206, China
}

ameimuxiaozi@126.com, ’kong.xiangyu1990@163.com, nanbu_huangfei@126.com

Keywords: large-scale wind base; sensitivity method; weakly chained diagonally dominant matrix; voltage fluctuation; quantitative voltage control

\begin{abstract}
The random variation of wind speed causes active power and reactive power injection of large-scale wind base to fluctuate constantly, this in turn leads to voltage fluctuation and unqualified voltage amplitudes of this area. This article proposes quantitative voltage control to solve this problem. Firstly, analyze the reason and characteristic of voltage fluctuation in large-scale wind base. Then apply sensitivity method to derive voltage/reactive power sensitivity coefficient matrix; this matrix is the product of two special matrices, one is symmetric and the other is diagonal. It can be demonstrated that the symmetric one has a relatively bigger value at the diagonal line; combined with the diagonal one, several simple conclusions can be obtained. According to the conclusions, quantitative voltage control flow can be established. In addition, operating mode of wind turbine generator is taken into account in the simulation process. Simulation results of IEEE 9 nodes system and an actual system demonstrate the validity of proposed measures.
\end{abstract}

\section{Introduction}

With the rapid development of wind power, integrating it into the power system has great effect on the system security and economic dispatch, causing series of problems. The reactive power and voltage problem is one of them, catching attention gradually [1]. Reference [2] studied reactive power regulation ability of doubly-fed induction generators. Reference [3] investigated the impacts of DFIG operation modes on system voltage stability and pointed out that constant-voltage control has a greater advantage than constant-power factor control on improving the system voltage stability by taking full advantages of DFIG's reactive power regulation ability. However, considering that DFIG may work in constant power factor mode and reactive power regulation of all DFIGs is complex and time-consuming, quantitative voltage control at some important nodes is necessary. This paper establishes quantitative voltage control process based on sensitivity coefficient; when voltages fluctuate because of wind speed variation, the voltage level of wind base can be maintained by guaranteeing the voltages at some key nodes qualified.

\section{Reason and characteristic of voltage fluctuation in large-scale wind base}

Voltage drop from high voltage bus of the wind farm to the infinite system bus can be written as follows:

$$
\Delta \mathrm{U}^{\prime}=\Delta \mathrm{U}+\mathrm{j} \delta \mathrm{U}=(\mathrm{PR}+\mathrm{QX}) \mathrm{U}_{1}+\mathrm{j}(\mathrm{PX}-\mathrm{QR}) \mathrm{U}_{1} .
$$

Where $P$ and $Q$ are active and reactive power injected into the system respectively; $\mathrm{U} 1$ is rated voltage; $R$ and $X$ are line parameters. Typically, the imaginary component of Eq. 1 can be ignored. The random variation of wind speed causes active power $P$ and reactive power $Q$ here to change and in turn lead to voltage fluctuation.

For distribution is quite dispersed, the wind farms cover a wide area. This makes geographically dispersed effect of wind farms distinct. Correspondingly, the time different wind farms reach the 
peaks and valleys of wind speed and the moment of the maximum output change rates appear, vary from each other, resulting in a difference in the time the maximum voltages emerge and the largest voltage variation [4].

\section{Sensitivity Matrix Calculation and Matrix Analysis}

Sensitivity matrix calculation. Because of the high ratio of $X / R$ in $\mathrm{AC}$ transmission lines, reactive power change is strongly related to bus voltage amplitude. And therefore $P Q$ decomposition conclusions in power flow calculation can be used and transformed:

$$
\Delta \mathrm{U}=\left(-\mathrm{B}^{\prime \prime}\right)^{-1} \mathrm{U}^{-1} \Delta \mathrm{Q}=\mathrm{S}_{\mathrm{UQ}} \Delta \mathrm{Q} .
$$

Where $\Delta Q$ and $\Delta U$ are the dimensional vector of reactive power injection deviation and voltage variation at each $P Q$ node respectively; $B^{\prime \prime}$ is the imaginary component of admittance matrix with slash node(nodes) and $P V$ node omitted, which is invertible and highly sparse; $U$ is the diagonal matrix made up of voltage amplitude at each $P Q$ node; $S_{U Q}$ is the sensitivity matrix.

Matrix analysis. Suppose $B^{\prime \prime}$ is an $N$ order matrix. Establish a new matrix as follows:

$$
\mathrm{A}=\left(-\mathrm{B}^{\prime \prime}\right)^{-1}=\left(a_{\mathrm{ij}}\right)_{\mathrm{N} * \mathrm{~N}} \text {. }
$$

When there are no phase shifters, $B^{\prime \prime}$ is a symmetric and weakly chained diagonally dominant $M$ matrix [5]. For a weakly chained diagonally dominant $M$ matrix, reference [5] points out that its diagonal elements satisfy Eq. 4.

$$
\begin{aligned}
& a_{\mathrm{ii}} \geq a_{\mathrm{ji}}, a_{\mathrm{ji}} \geq 0 \quad \mathrm{i}, \mathrm{j}=1,2 \ldots \mathrm{N} . \\
& \mathrm{A}=\mathrm{A}^{\mathrm{T}} .
\end{aligned}
$$

According to Eq. 5, Eq. 6 can be obtained.

$$
a_{\mathrm{ii}} \geq a_{\mathrm{ij}} \quad \mathrm{i}, \mathrm{j}=1,2 \ldots \mathrm{N} \text {. }
$$

Thus, no matter in the row or column it is located, $a_{\mathrm{ii}}$ has the largest value, which is of guiding significance.

$$
\Delta \mathrm{U}_{\mathrm{i}}=\Delta \mathrm{Q}_{1} * a_{\mathrm{i} \gamma} \mathrm{U}_{1}+\Delta \mathrm{Q}_{2} * a_{\mathrm{i} 2} \mathrm{U}_{2}+\ldots+\Delta \mathrm{Q}_{\mathrm{i}} * a_{\mathrm{i} i} \mathrm{U}_{\mathrm{i}}+\ldots+\Delta \mathrm{Q}_{\mathrm{N} *} a_{\mathrm{iN}} \mathrm{U}_{\mathrm{N}} \quad \mathrm{i}=1,2 \ldots \mathrm{N} .
$$

Consider voltages in per unit are near 1, following conclusions can be drawn from Eq. 7:

(1) Reactive power compensation at certain node can not only change the node voltage itself, but also to some extent change voltage level of the system. By increasing the injected reactive power at a node, its own voltage and the system voltage level will both be improved. Correspondingly, its own voltage and the system voltage level will both be decreased by decreasing the injected reactive power at a node.

(2)As $a_{\mathrm{ii}}$ has the largest value in the row and column it is located, reactive power regulation at node $i$ works efficiently and economically. From the angle of column, this compensation contributes most to voltage change of node $i$; from the angle of row, least compensation is needed at node $i$ to compensate the voltage deviation. Suppose voltage at node $i$ is out of limit. If one adjusts reactive power injection at node $\mathrm{j}(\mathrm{j} \neq \mathrm{i})$, the largest voltage change will appear at node $\mathrm{j}$ and it is likely that voltages at node $\mathrm{j}$ and other nodes are out of limit, which will lead to an increase in the difficulty of regulation. The suggested way should be: give priority to reactive power adjustment at the out of limit node; if there is no reactive power regulation device or reactive power regulation capacity is used up, one should in turn adjust reactive power at node which has the largest sensitivity coefficient other than the one (ones) has (have) no regulation ability anymore, until all voltages are qualified. 
(3)Generally, node voltages are out of their low limits, thus reactive power compensation at such nodes will be more sensitive.

\section{Quantitative Voltage Control of Large-scale Wind Base}

Voltage control goal in this article is to quickly seek a group of control measures, which can ensure all regional voltages qualified with less action times. Here qualified voltage amplitude is between 0.97 and 1.07 in per unit. Flow graph of voltage control can be obtained according to the conclusions above, as depicted in Fig.1.

\section{Calculating Cases and Results Analysis}

Calculating Cases. Two cases are prepared. The first case (case1) is IEEE 9 nodes system, with a synchronous generator at node 2 replaced by a $321 \mathrm{MW}$ wind farm. The second case (case2) is an actual system with 17 wind farms, with a total installed capacity of 3516 MW. 16 of them is 201 MW. They are connected to grid by substation 1 to substation 7. Details can be seen from the wiring diagram in Fig.2. Suppose all the wind machines are DFIGs. Consider the different operating mode of DFIG [3] and increase active power output of the wind farms, put down the results of voltage condition with and without quantitative voltage control respectively.

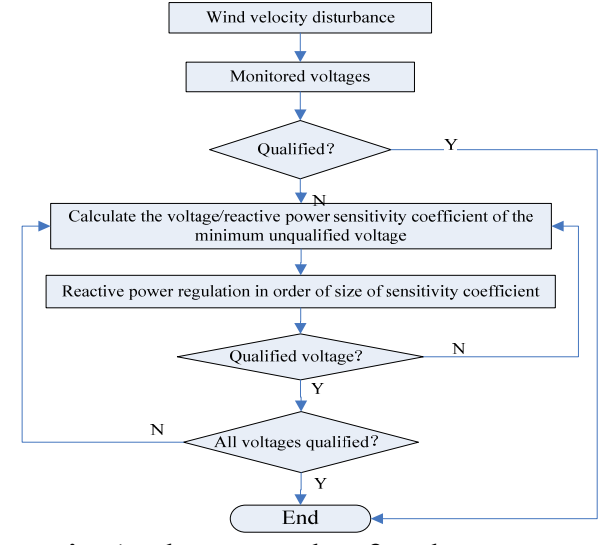

Fig. 1 Flow graph of voltage control

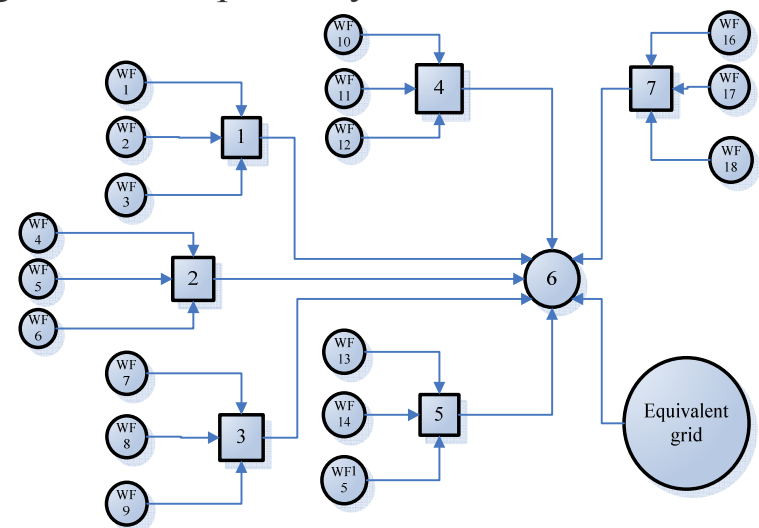

Fig. 2 Wiring diagram of case 2

Results analysis. Simulation results of case1 and case 2 indicates that operation mode of DFIG has a significant effect on voltage level of large-scale wind base, constant voltage mode (CV in short and $\mathrm{V}=1.0$ ) of DFIG can excellently lessen the influence of random variation of wind speed on voltage level at wind base area, as depicted in Fig.4 and Fig.6.

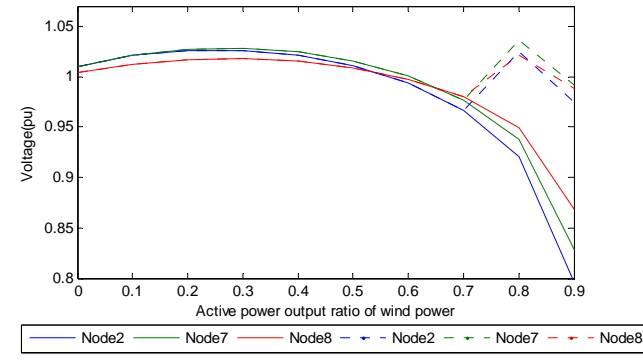

Fig.3 Voltage simulation curves in $\mathrm{CP}$ mode(case1)

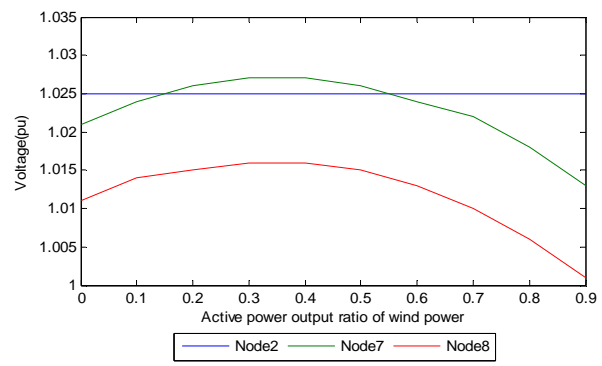

Fig.4 Voltage simulation curves in CV mode(case1) 


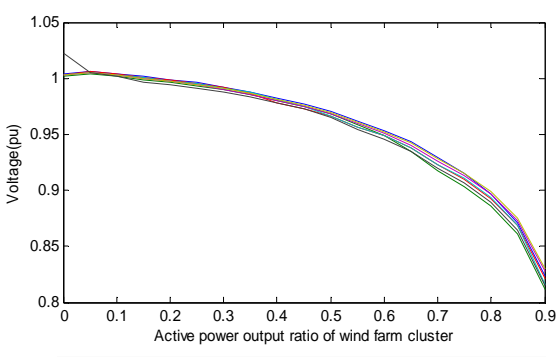

$-1-2-3-4-5-6$

simulation curves in $\mathrm{CP}$
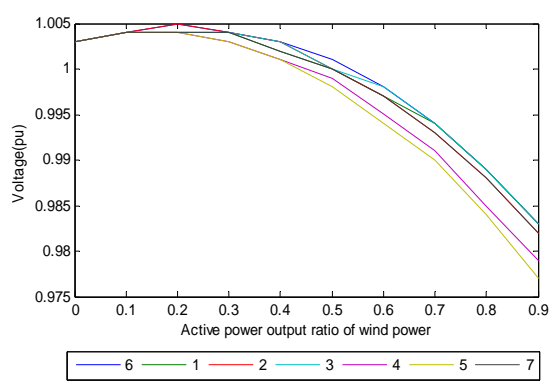

Fig.5 Voltage

mode without voltage control(case2)

Fig.6 Voltage Simulation curves

in CV mode(case2)

However, when constant power factor (CP in short and power factor is equal to 1) mode is adopted, voltages of wind base will suffer from a relatively larger influence of wind speed variation. This can be seen from Fig.3 and Fig.5. In Fig.3, when active power output ratio reaches about 75\%, monitored voltage at node 2 becomes unqualified; in Fig.5, this ratio is about $45 \%$ and the unqualified node is node 7. Dash curves of Fig.3 and Fig.7 give the voltage curves after quantitative voltage control has been implemented, all the monitored voltages are qualified throughout the likely increasing active power output of wind base. The results demonstrate the effectiveness of the proposed measures.

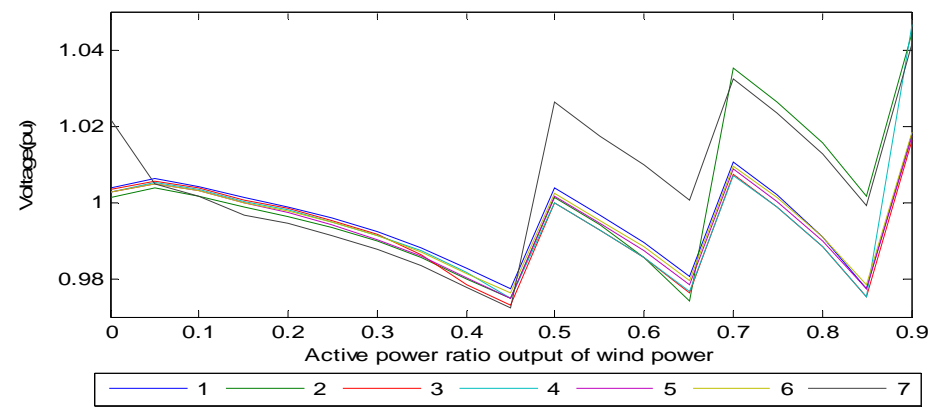

Fig.7 Voltage simulation curves in $\mathrm{CP}$ mode with voltage control(case2)

\section{Summary}

The random variation of wind speed causes active power and reactive power of wind base to fluctuate constantly, this in turn leads to voltage fluctuations.

Constant voltage mode of DFIG can excellently lessen the influence of random variation of wind speed on voltage level, while constant power factor mode suffers from a relatively larger influence of wind speed variation.

Based on sensitivity coefficient matrix, reactive power regulation can be efficient and economical. By improving voltage level of monitored nodes, voltages in the wind base area can be well maintained.

\section{References}

[1]Xuefang Liu, Hongtao Wang, Huadong Sun, Area Automatic Voltage Control of Large-Scale Wind Farms, Power and Energy Engineering Conference (APPEEC), 2012 Asia-Pacific, pp.1-4, 27-29, March 2012.

[2]Mustafa Kayikci and V.Milanovic, Reactive power control strategies for DFIG-based plants, IEEE transactions on energy conversion, Vol.22, No.2, pp. 389-396, June 2007.

[3]Ming Ding, Binbin Li, Pingping Han , Impacts of doubly-fed wind turbine generator operation mode on system voltage stability, Power Electronics for Distributed Generation Systems (PEDG), 2010 2nd IEEE International Symposium on, pp.602-606, 16-18 June 2010. 
[4]Bo Du, Wenying Liu, Ningbo Wang, etal, Voltage Optimization Control of the Region Integrating with Large Scale Wind Power Based on Sensitivity Method, Electric Power Science and Engineering, Vol.28, No.8,pp.13-17,August 2012.

[5]Shuzhen Pan, Shencan Chen, An upper bound for $\left\|\mathrm{A}^{-1}\right\|_{\infty}$ of weakly chained diagonally dominant matrices, Journal of Fuzhou University,Vol.40, No.3, pp.281-284, June 2012. 